\title{
Evaluation of Effect of Oral Microorganism on Surface Topography of Arylic Resin Denture Base Materialby Three Dimensional Profilometry Using Laser Triangulation Sensor- In Vitro Study
}

\author{
Dr. Mukesh Soni*, Mds, Dr Deshraj Jain ${ }^{* *}$, Mds \\ * ASSOCIATE PROFESSOR, Department of Prosthodontics, Government College of Dentistry, Indore \\ ** PROFESSOR AND HEAD, Department of Prosthodontics, Government College of Dentistry, Indore
}

\begin{abstract}
:
Purpose: The aim of this study was to evaluate the effect of oral microorganism on surface topography of acrylic resin denture base material.

Materials and methods: Fifty rectangular plates of dimensions $30 \mathrm{~mm} \times 20 \mathrm{~mm} \times 1 \mathrm{~mm}$ were fabricated with heat polymerizing acrylic resin denture base material. an area of $10 \mathrm{~mm} \times 10 \mathrm{~mm}$ in the center of each acrylic plate was marked for evaluation of surface topography by Laser Triangulation Sensor.The plates were then sterilized by immersion in $2 \%$ Gluteraldehyde for 30 minutes, and placed in sterilized containers. saliva sample were collected from 40 healthy edentulous people of 40 to 60 years in age wearing acrylic complete denture. 0.1 $\mathrm{ml}$ of saliva was immediately poured on central portion of each were placed in incubator at 37 degree centigrade for $72 \mathrm{hr}$. after $72 \mathrm{hr}$ each plate was inspected for bacterial colonies.

Results: The mean value of difference of mean surface roughness value ( $\mathrm{ra}$ ) of acrylic plates before culture and after culture of microorganism is 2.13343 with overall standard deviation of 0.9964, and standard error 0.1821.

Conclusion: The acrylic resin plates showed some amount of roughness even after polishing (mean surface roughness value ' $R a$ ') before culture of microorganism on them. The acrylic resin plate showed an increase in mean surface roughness after culture of microorganism. Thus this roughness will deteriorate the denture by the time, even after the use of latest denture cleanser and other auxiliaries, by offering more plaque and microorganism accumulation and cycle go on.
\end{abstract}

Keywords: Surface topography, microorganism, laser triangulation sensor.

\section{Introduction}

The establishment of smooth surface has always been prime objective for denture prosthesis because of the biologic consequence of plaque accumulation ${ }^{1 .}$ The process by which dentures accumulate plaque, stain and calculus is apparently similar to that process which takes place on natural teeth ${ }^{2}$.

Esthetic consideration aside, the layer of the plaque, which forms on the tissue-fitting surface of the denture, is probably of greatest clinical significance. A variety of soft tissue changes are associated with dentures. These changes manifest themselves as a series of related symptom complexes which includes denture stomatitis, inflammatory papillary hyperplasia and chronic candidiasis. Theilade ${ }^{2}$ suggests that the presence of denture plaque constitutes the principal cause leading to the inflammation of palatal mucosa.

Denture has an environment that encourages the residence of aerobic, facultative and anaerobic micro flora. The ability of microorganisms to adhere to surface exposed to the flushing action of fluids is a prerequisite for successful colonization. Lactobacillus among the coccus groups is an acidogenic and aciduric groupthat produces acid by fermentation of food debris entrapped in the denture, and can survive in acidic media ${ }^{3,4,5,6,7}$

The acid produced may etch the polished surfaces of the denture producing rough surfaces. These rough surfaces will offer more accumulation of plaque, which in turn will provide room for residing microbes and thus more microbes will grow and this cycle goes on. Scratches on tissue bearing surfaces will not only make it more difficult to maintained cleanliness but might also became a source of tissue irritation; in severe cases the actual adaptation of the denture may be impaired.

This study was carried out to evaluate the surface changes produced by predominant microbes of oral cavity on acrylic resin denture base material. The surface changes were measured with the help of profilometry using three dimensional laser triangulation sensor.

\section{I . Materials And Method}

50 rectangular plates of dimensions $30 \mathrm{~mm} \times 20 \mathrm{~mm} \times 1 \mathrm{~mm}$ were fabricated with heat polymerizing acrylic resin denture base material. An Area of $10 \mathrm{~mm} \times 10 \mathrm{~mm}$ in the center of each acrylic plate was marked for 
evaluation of surface topography by laser triangulation sensor. The acrylic plate was held in a special fabricated metallic device, and mounted on translation platform with the help of screw. Translation platform was set to a predefined initial position along $\mathrm{X}$-axis and $\mathrm{Y}$-axis. The initial position was determined as one corner of area of interest. The initial position of each acrylic plate along $\mathrm{X}$-axis and $\mathrm{Y}$-axis was noted so that plates can be replaced on the same position relative to translation stage.

Translation platform was then moved along with the target in $\mathrm{X}$ and $\mathrm{Y}$ direction in defined step sizes. The image definition used in this study was twenty profiles (X step of 500 microns) and each profile consisting of 20 points (Y steps of 500 microns). The laser triangulation sensor calculated the depth of the plate specimen in $\mathrm{Z}$ direction in 3- dimensional view for each step. Thus the surface profile of the pre determined $10 \mathrm{~mm} \mathrm{x}$ $10 \mathrm{~mm}$ area was recorded.

The complete data i.e. the $\mathrm{X}$ and $\mathrm{Y}$ position along with their corresponding depth was then communicated to the computer. The computer processed the data and plotted a three dimensional graph corresponding to the surface texture of the given area. The process was repeated for all 50 plates.

The plates were then sterilized by immersion in $2 \%$ Gluteraldehyde for 30 minutes, and placed in sterilized containers. Nutrient agar mixed with Blood agar culture media at 45 degree ${ }^{\circ} \mathrm{C}$ was poured on each plate. The plates were kept at room temperature till the media solidified, then stored in refrigerator till use.

Saliva sample were collected from 40 healthy edentulous people of 40 to 60 years in age wearing acrylic complete denture, in 40 different plastic containers with the help of sterilized disposable syringes and labeled for the respective plates. $0.1 \mathrm{ml}$ of saliva was immediately poured on central portion of each sterilized Acrylic plate. These plates- with specimens and control plates without specimen were placed in incubator at 37 degree centigrade for $72 \mathrm{hr}$.

After $72 \mathrm{hr}$ each plate was inspected for bacterial colonies. Out of 40 plates 30 plates showed well formed colonies, 3 plates showed non specific colonies, and 7 plates were devoid of any colonies. So these 10 plates were discarded. These 30 culture plates with well developed colonies of bacterial growth were chosen for further study. These colonies were identified by gram staining and further biochemical reaction and identified as Streptococcus mutans and Candida albicans. 30 minutes.

All these Acrylic plates were washed under running tap water and sterilized by $2 \%$ Gluteraldehyde for

The Acrylic plates were mapped in the same way taking care of the initial position. Same procedure was applied for all thirty acrylic plates before and after culture of microorganism.

\section{Observations}

Table I - Showing statistical analysis of data collected after evaluating the surface topography (mean surface roughness ' $\mathrm{Ra}$ ') of specimens before culture and after culture of microorganism on them).

Table II - Showing statistical analysis of data collected after evaluating the surface topography (mean of difference surface roughness ' $\mathrm{Ra}$ ') changes of specimens before culture and after culture of microorganism on them).

The data was statistically analyzed for difference in surface topography before and after culture of microorganism (Fig. 1). The measurement was based on 500 microns resolution and each X and Y runs in 20 steps to measure total $10 \mathrm{x} 10 \mathrm{~mm}$ area of the plate. The measuring conditions were as follows. Acrylic plates moved 1 step in $\mathrm{x}$ direction, and 20 steps in y direction, so for 20 steps in $\mathrm{x}$ direction, we got total of 400 readings (steps). The three dimensional digital image was produced on a graph for each acrylic plate before and after culture.

Laser Triangulation Sensor measure roughness by maximum peak to minimum valley height in micrometer. From 20 steps the mean average of distance between any 3 maximum peaks to minimum valley will reveal the average roughness of that sample specimen acrylic plate.

The statistical analysis was computed by Meanwhich was calculated after evaluating the surface topography (mean surface roughness 'Ra') of acrylic plates before culture (Fig. 2) and after culture (Fig. 3) of microorganism on them for all the samples andPaired ' $t$ ' test for Dependent Samples.

\section{Results}

The mean surface roughness value (Ra) of acrylic plates before culture of microorganism is 0.264 with standard error of 0.1821. (Table II, III)

2. The mean surface roughness value (Ra) of acrylic plates after culture of microorganism is 2.434 with standard error of 0.1821. (Table II, III)

3. The mean value of difference of mean surface roughness value $(\mathrm{Ra})$ of acrylic plates before culture and after culture of microorganism is 2.13343 with overall standard deviation of 0.9964, and standard error 0.1821. (Table III) 
4. The value of ' $t$ ' in t-test statistics was calculated to be 2.732 significant at $5 \%$ level of significance. (Table III)

\section{Discussion}

There are various reason for surface changes of acrylic complete denture when worn in the oral cavity, such as, method of cleansing denture, type of denture cleansing solution, poor quality of polishing at the time of insertion of denture, chemical effect of salivary ingredient and poor oral hygiene that leads to accumulation of salivary micro flora and plaque, food habits, rough handling of denture, Although acrylic resin when used in the form of denture base material for complete denture prosthesis provide acceptable esthetics, but roughness produced on the surface has been a matter of serious concern. ${ }^{8,9}$

Both in-vivo and in-vitro studies, regardless of simplicity or complexity of machinery or measurement method have constantly demonstrate the accumulation of plaque on denture surface. , $^{2,8,8,10,11,12}$

The process by which denture accumulates plaque stain and calculus is apparently similar to that process which takes place on natural tooth surface. ${ }^{13}$ Denture hygiene is an important element to maintained smooth surface hygiene of denture base resin, which always has been a neglected aspect.

Gibson and Houte ${ }^{14}$ in 1973 have defined dental plaque as dense, non-calcified bacterial masses so firmly adherent to the tooth surface, that they resist wash off by salivary flow and rinsing. The initial stage in plaque formation involves attachment of microorganism to the pellicle surface, followed by adherence of bacteria to each other, mediated by the component of the plaque matrix, causing the microorganism to coalesce and form large bacterial masses.

0.5-1.5 micron thick salivary pellicle is deposited on every surface in the oral cavity, natural or synthetic, ${ }^{9}$ which in turn provides a substrate to which oral debris (such as mucin, food particles and desquamated epithelial cells) and microorganisms (bacteria and fungi) readily adhere. Certain adherent bacteria and fungi convert materials such as sucrose and glucose in the oral environment into a protective plaque covering under which they can thrive and proliferate further.

Adherence of microorganisms and debris is also favoured by rough or otherwise irregular surface topography. ${ }^{9,10,15}$ Surface irregularities provide an increase in surface area and an expansion in the number of niches not readily cleansed by actions of the tongue or other orofacial musculature. This is a particular concern in the case of oral appliances fabricated out of acrylic resin. Despite an outwardly smooth appearance, these appliances have a pockmarked surface when viewed under microscopic magnification. This is due to bubble formation from unpolymerized monomer in the course of denture processing

The fungal organisms that are most commonly associated with denture plaque are of the genus Candida. ${ }^{14,16,17,18}$ these yeasts are present in the saliva of a majority of denture wearers and display an affinity for adherence to acrylic resin. They not only hamper the smoothness of acrylic surface but also responsible for denture stomatitis, Cahn 1936, Callar 1945. The most prevalent and predominant bacteria associated with denture wearer is Streptococcus and Lactobacilli group that are present in plaque. ${ }^{15,19}$

The role of bacterial plaque in acid production has been studied intensively by Etherington, Fosdick, Hanke, Hardwick, Miller, Stephan and Strafford. The fate of acid produced by mouth bacteria as it combines with the saliva on one side and the tooth on the other is not known. Hill, Manly, Tular and Wach have performed ingenious in vitro experiments on this problem. ${ }^{3}$ In 1954 Nolte and Sumter studied the change in $\mathrm{pH}$ of dental plaque material and surrounding saliva. ${ }^{3}$

J. S. Landa ${ }^{10}$ in 1960 discussed factors of oral hygiene, chemicotoxicity, Nutrition, Allergy and conductivity. He conclude rough surface of dentures as well as surface porosity of denture base materials provide a favorable sites for harboring bacteria.

The main acid production in saliva is lactic acid. ${ }^{20}$ The hypothesis is that this may alter the surface of acrylic resin in the form of roughness. Thus this roughness offer more accumulation of plaque and this cycles goes on.

This study was taken up to evaluate in-vitro the effect of oral microflora on surface topography of acrylic resin denture base by three dimensional profilometry using laser triangulation sensor. In the present invitro study an attempt was made to find out, if there exist an effect of oral microorganism on surface topography of acrylic resin denture base. Acrylic resin plate $30 \times 20 \times 1 \mathrm{~mm}$ in dimensions was chosen in the study, ${ }^{15}$ because of the fact that, microorganism can grow on teeth as well as on denture surface, and they do not require nutrients from them. So also plates are easy to prepare and less expensive.

A total of 40 edentulous subjects wearing complete denture were randomly selected for saliva sample collection. The subjects were chosen from different socioeconomic strata, namely 2 from higher, 22 from middle and 16 from low socioeconomic strata. Subjects were chosen such that, who are free from habits of pan tobacco chewing or smoking. This shows that subjects were selected irrespective of the above mentioned factors, so that they represented the population to which they belonged. Only those subjects who were free from 
any oral or systemic disease were chosen. This was done in order to avoid any effect on the results of the study, due to these factors.

Control group consists of 10 Acrylic plates of same material and size. They are constantly present in environment of experimental group throughout the study, except inoculation of saliva and culture of microorganism on them. This was done in order to rule out of any other factors, such as, sterilization procedure of acrylic plates, handling, washing etc. which can affect the surface of resin. This study shows that the surface of control group remains the same thought the study.

Surface roughness assessment has been made in various studies ${ }^{1,21}$ by measuring loss of weight, luster change, microscopic scanning and profilometry. In the present study surface topography of acrylic specimens is measured as surface changes in the form of roughness by three- dimensional laser profilometry before and after culture of microorganism on acrylic plates. The main advantage of this method to other is that, it is more accurate, easy to conduct and provide a three dimensional graph of the surface topography of the material of interest, so that mean surface roughness (Ra) of acrylic plates can be easily calculated.

Result shows that mean value of surface roughness (Ra) of acrylic plates before culture of microorganism was 0.264 microns with standard deviation 0.16 . The mean value of roughness of acrylic plates after culture of microorganism was 2.434 microns with standard deviation of 0.17 . The mean of difference before and after culture of microorganism on acrylic plates is 2.13343 with standard deviation of 0.9964 and standard error of 0.1821 . The calculated value of ' $t$ ' in t-test of difference of means of surface roughness before and after culture of microorganism on acrylic plates was calculated to be 2.732 which is more than the critical value 2.03 at $5 \%$ level of significance. Thus, we reject the null hypothesis $\left(\mathrm{H}_{0}\right)$, which states that means are equal; hence it lies in rejected region. We concluded that there is significant difference in the studies before and after culture.

Since there was paucity of such type of studies, so result can not be correlate with the other studies, but another similar studies in support of our result was by Morgan T.D. and Wilson M who found that there was a direct correlation of acrylic resin surface roughness and adherence of bacteria on them but after $4 \mathrm{hr}$ incubation surface roughness appeared to have no effect on number of adherent bacteria.

In the present study a Streptococcal flora was found on the surface of acrylic. Similar physiological varieties of Streptococcus were found on the dentures as previously describe from dental plaque (Carlson, 1965, 1967a, 1968a). ${ }^{15}$

\section{Conclusions}

1. The acrylic resin plates showed some amount of roughness even after polishing (mean surface roughness value ' $\mathrm{Ra}$ ') before culture was 0.264 .

2. The acrylic resin plate showed an increase in mean surface roughness after culture of microorganism (2.434).

3. There was significant difference in mean surface roughness value (surface topography 'Ra') of acrylic plates (2.13343) before culture and after culture of microorganism on them. And that roughness will deteriorate the denture by the time even after the use of denture cleanser and other auxiliaries, because denture remain in oral cavity that is the favorable fermented area for microbes more than $12 \mathrm{hr}$ per day.

\section{Bibliography}

[1]. E. Barastegguai. Surface roughness of finished composite resins. JPD. Nov. 1992.

[2]. Abelson DC. Denture plaque \& denture cleansers. JPD, 45[4]:376-379, April 1981.

[3]. WM Nolte. In vitro changes in the $\mathrm{pH}$ of dental plaque material and surrounding saliva.

[4]. William Nolte. Oral microflora. Chapter 9:198-237

[5]. D. Orastavik. The in vitro attachment of an oral streptococcus sp. to the acquiredpellicle. Archives Oral Biology. 23:167-173, 1978.

[6]. Gordon E. Green and Mathew C. Dodd. A study of the bacterial flora of caries-susceptible and caries-immune saliva: J.D. Res. August 1956.

[7]. Shafer Hyne Levi. Dental caries. Textbook of Oral Pathology.

[8]. Kennedy Shay. Denture hygiene: A review update I.

[9]. Kennedy Shay. Denture hygiene: A review update II Denture debris.

[10]. Landa JS. Trouble shooting in complete denture prosthesis part VI factors of oral hygiene chemicotoxicity, Nutrition Allergy \& conductivity. JPD. 10[5]:887-890, Sept 1960.

[11]. Reenen Van. Microbiologic studies on denture stomatitis. J Pros Dent. 30:493-505, 1973.

[12]. Tarbet WJ. Denture plague, quiet destroyer. J Pros Dent. 48:647, 1982.

[13]. Edgerton M, Levine M. Characterization of acquired denture pellicle from healthy and stomatitis patients. JPD. 68[4]:683-691, 1992.

[14]. Gibbons P \& Houte K. On the formation of dental plaque. Journal of Periodontology. 144 : 347, June, 1973

[15]. J. Carlson. Prevalence of streptococcus sanguis and s. mutans in the mouth of person wearing full denture. Archives Oral Biology. 14:243-249, 1969.

[16]. Davenport JC. The oral distribution of candida in denture stomatitis. BDJ. 129:151, 1970.

[17]. Nater Groenman. Etiological factor in denture mouth syndrome. JPD. 40:367, 1978.

[18]. Anthony M. Iacopino, William F. Wathen. Oral candidal infection and denture stomatitis: A comprehensive review. 
[19]. Morgan and Wilson. The effect of surface roughness and type of denture acrylic on biofilm formation by streptococcus in constant depth film fermenter. J Applied Microbiology. 91: 47-53, July 2001.

[20]. JJ. Ray. Lactic acid production in saliva. Archives Oral Biology. Jan 1956

[21]. Sexon J. C. \& Ralph W. Philips. Studies on the effect of abrasives on acrylic resin.

Figures and Tables

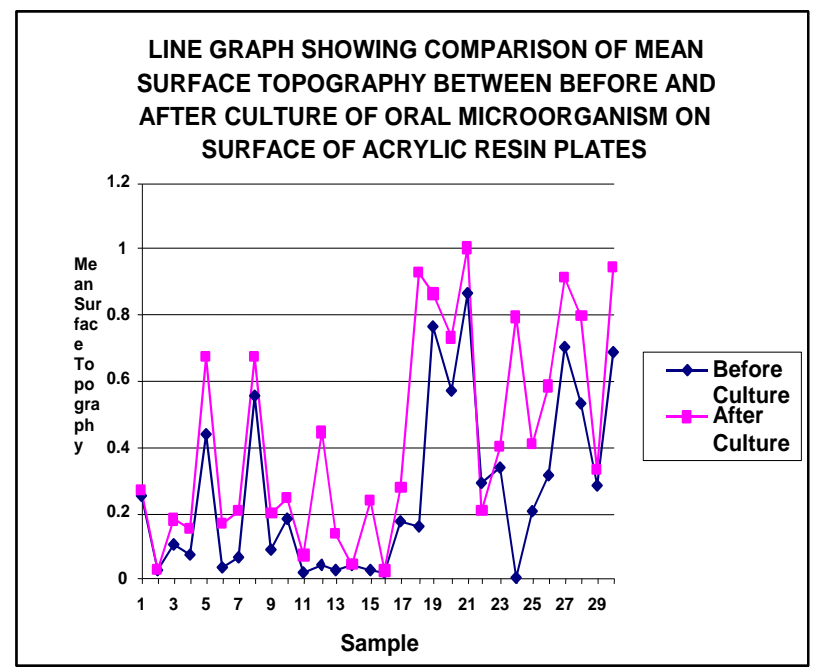

Fig 1

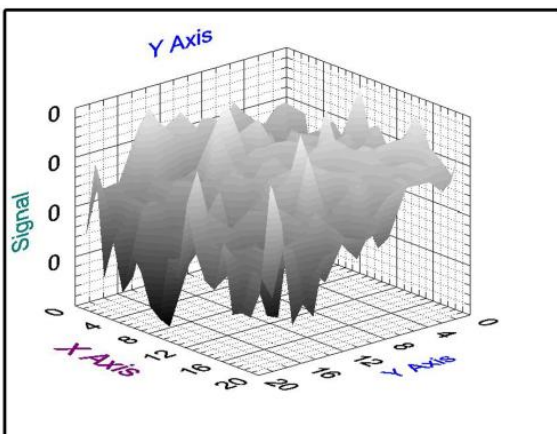

Fig.2

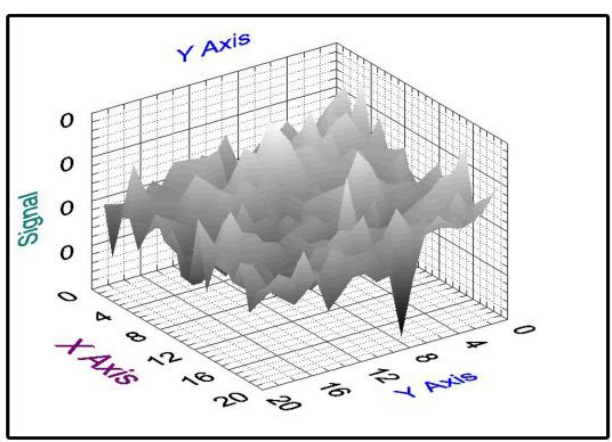

Fig. 3

\begin{tabular}{|l|l|l|}
\hline & $\begin{array}{l}\text { Before culture of microorganism on } \\
\text { Acrylic plate. }\end{array}$ & $\begin{array}{l}\text { After culture of microorganism on } \\
\text { Acrylic plate. }\end{array}$ \\
\hline N & 30 & 30 \\
\hline Mean & 0.264 & 2.434 \\
\hline S.D. & 0.16 & 0.17 \\
\hline
\end{tabular}

Table 1

\begin{tabular}{|l|l|}
\hline Mean of Difference & 2.13343 \\
\hline S.D. & 0.9964 \\
\hline S.E. & 0.182 \\
\hline Test statistic 't' & 2.732 \\
\hline Instrument error & 10 microns \\
\hline
\end{tabular}

Table 2 